\title{
Interference Suppression in Heterogeneous Massive MIMO Systems with Imperfect CSI
}

\section{Rana Sedghi}

Sahand University of Technology

masoumeh azghani ( $\nabla$ elmaazghani@yahoo.com )

Sahand University of Technology https://orcid.org/0000-0003-0043-9913

\section{Research Article}

Keywords: Massive MIMO system, Heterogeneous networks (HetNet), Interference suppression, Interference management

Posted Date: December 21st, 2021

DOI: https://doi.org/10.21203/rs.3.rs-1114129/v1

License: (1) This work is licensed under a Creative Commons Attribution 4.0 International License. Read Full License 


\title{
Interference Suppression in Heterogeneous Massive MIMO Systems with Imperfect CSI
}

\author{
Rana Sedghi ${ }^{\mathrm{a}}$, Masoumeh Azghani ${ }^{\mathrm{a}, *}$ \\ ${ }^{a}$ Laboratory of Wireless Communication and Signal Processing (WCSP), Faculty of \\ Electrical Engineering, Sahand University of Technology, Iran.
}

\begin{abstract}
Interference management is of paramount importance in heterogeneous massive mimo networks (HetNet). In this paper, an algorithm has been suggested to suppress the interference in large-MIMO HetNets with imperfect channel state information(CSI). The proposed technique controls both the intra-tier and cross-tier interference of the macrocell as well as the small cells. The intra-tier interference of the macrocell as well as the cross-tier interference have been minimized under maximum transmission power and minimum signal to interference and noise ratio (SINR) constraint. The channel estimation error matrix has also been modeled using the joint sparsity property. The precoding algorithm is thus achieved through the application of semi-definite relaxation and block coordinate descent techniques. The intra-tier interference of the small cells are addressed with the aid of the zero forcing scheme. The proposed method has been validated through various simulations which confirm the superiority of the algorithm over its counterparts.
\end{abstract}

Keywords: Massive MIMO system, Heterogeneous networks (HetNet), Interference suppression, Interference management.

\footnotetext{
* Corresponding author

Email address: mazghani@sut.ac.ir (Masoumeh Azghani)
} 


\section{INTRODUCTION}

Due to the increasing number of users and data traffic, the massive MIMO systems as well as the heterogeneous network (HetNet) have emerged as proper solutions to achieve reliable communication, spectral efficiency and energy effi-

5 ciency in the next generation wireless cellular networks [1]. The advantages of massive MIMO systems and HetNet technology include increasing the transmission rate, and improving the channel capacity and cell coverage using a large number of antennas and small cells. In HetNets, the usage of multiple small cells across a macro cell with lower transmit power improves the quality of service at the same time of reducing the total power consumption significantly. The interference and energy efficiency (EE) are two important factors in these systems which should be handled in order to gain the aforementioned benefits. The small base stations (SBS) and macro base stations (MBS) share the frequency resource which leads to the cross-tier interference [2. Various techniques have been suggested in the literature to solve this issue. The interference coordination in HetNets has been accomplished by uplink power control and cell radius extension in [3. In [4, 5], interference alignment has been used as an efficient method to manage the inter-layer interference. Using nested arrays is an effective way to achieve high degrees of freedom (DOF) which enables the BS to remove its interference to the neighboring users [6].

The trade-off between inter-cell interference cancellation and sum-rate maximization has been modeled as an integer programing problem in [7, 6]. Due to the lack of instantaneous channel information from MBS to small users, statistical channel information has been used in [8] to design the minimum mean square error (MMSE) precoder for interference elimination. In $[9$, by utilizing the DOF provided by MBS, the corresponding signal of small users have been precoded in the null space of MBS to avoid interference. In [10], the interference mitigation and load balancing have been achieved by optimizing the precoding matrix, user association and scheduling, and power allocation. Another way for interference cancellation is to identify the small users which are severely suffer- 
ing from the MBS interference and eliminate their corresponding interference at the time of designing the MBS precoding matrix [11. The cooperative interference mitigation in HetNet based on almost blank sub-frame is considered in [2]. In [12], a resource allocation scheme has been provided using game-theory that takes into account both the energy efficiency and interference reduction in the presence of inaccurate channel state information in HetNets. Improving the energy efficiency by minimizing the total power consumption and designing the optimal precoder matrix is suggested in [13]. The energy efficient zero forcing (ZF) precoder for coordinated multi-point transmission of HetNets has been considered in [14. Energy efficiency maximization is achieved by deactivating some of the small cells using the Dinkelbach method in[15]. Another energy efficiency maximization algorithm has been suggested in [15] where the complexity of the optimization problem has been decreased. In [16], the beamforming matrix is designed to optimize the network energy efficiency under the 45 constraints of quality of service and power allocation. The compromise between spectral efficiency and energy efficiency has been established in [17 with capacity-constrained backhaul links. In [18], the ZF precoding and block diagonalization (BD) schemes have been used in the MBS and SBS, respectively, to reduce the downlink interference. In [19, the sparse precoding matrix is designed to suppress the interference and improve the energy efficiency in homogeneous networks in the presence of channel estimation error. A signal is called sparse where most of its entries are zero in some domain [20].

The motivation of this paper is to present an algorithm for interference suppression in massive MIMO HetNet with imperfect channel state information. constraints to guarantee the minimum signal to interference and noise ratio (SINR) and maximum transmission power for each of the users. The channel estimation error has also been modeled in the suggested problem with the joint sparsity term. This is based on the assumption that only a few of the user

60 channels have considerable channel estimation error. Using the semidefinite relaxation, the problem has been converted to a convex problem which is solved 


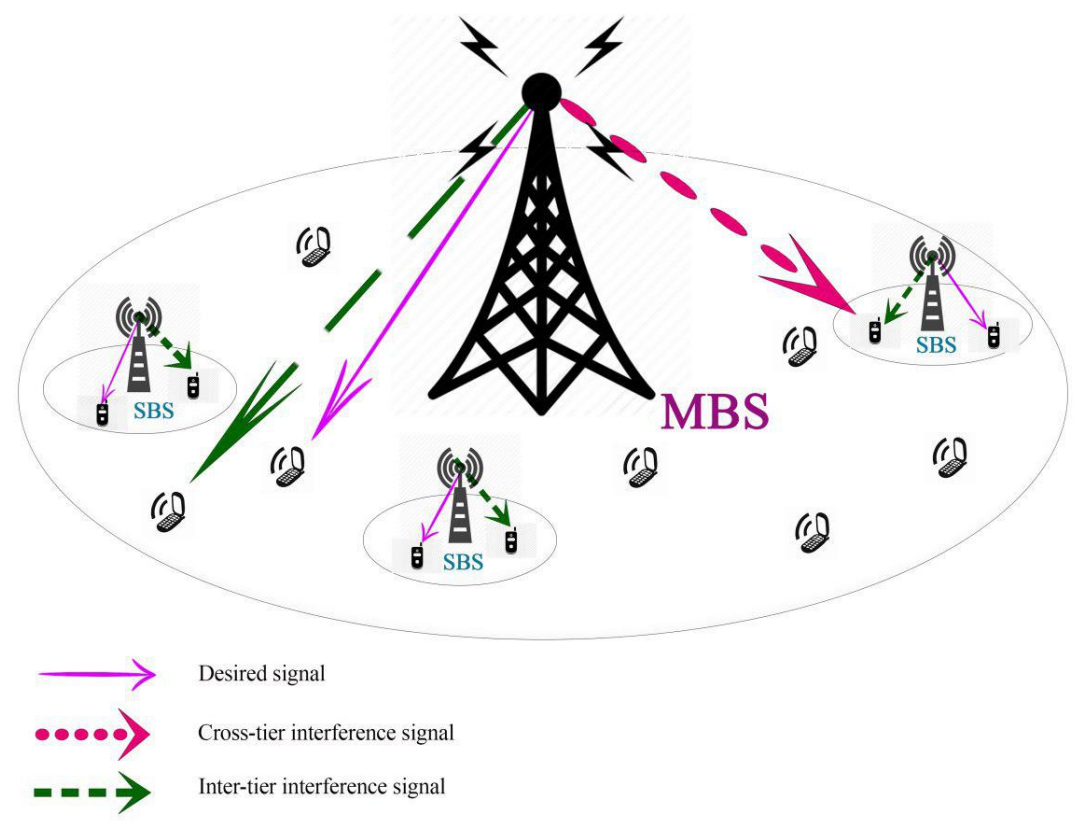

Figure 1: Downlink scenario for massive MIMO heterogeneous network.

with the aid of the block coordinate descent scheme. The simulation results have confirmed the outperformance of the algorithm from the perespective of energy efficieny and sum-rate. The rest of the paper is organized as follows: Section

65 II describes the system model. Section III introduces the proposed interference management scheme. The simulation results have been given in Section V and the paper is concluded in Section VI.

\section{SYSTEM MODEL}

A heterogeneous massive MIMO network with downlink scenario is considered with one MBS and $S$ SBSs which are randomly deployed in the macro cell as shown in Fig.1. The MBS uses a very large array of $N_{m}$ antennas to serve $M$ macro users (MU) that are randomly distributed in the macrocell. Each SBS with $N_{s}$ antennas only transmits data to the $K$ small users (SU) located in its coverage area. Also, all the users are single antenna. In the downlink data 
transmission mode, the received signal in the $m^{\text {th }} \mathrm{MU}$ is represented as:

$$
y_{m}^{M}=\left(\mathbf{h}_{m}^{M}\right)^{H} \mathbf{v}_{m}^{M} x_{m}^{M}+\sum_{\substack{l=1 \\ l \neq m}}^{M}\left(\mathbf{h}_{m}^{M}\right)^{H} \mathbf{v}_{l}^{M} x_{l}^{M}+n_{m}
$$

$x_{l}^{M}$ is the transmission signal from the MBS to the $l^{\text {th }} \mathrm{MU}$ and $\mathbf{v}_{l}^{M} \in C^{N_{m} \times 1}$ is the corresponding precoding vector. $\mathbf{h}_{m}^{M} \in C^{N_{m} \times 1}$ is the channel vector from the MBS to the $m^{t h}$ MU. $n_{m} \sim C N\left(0, \sigma_{m}^{2}\right)$ denotes the additive white Gaussian noise at the $m^{t h} \mathrm{MU}$. The second term in (1) represents the interference from the MBS received by the MU. In addition, since the SBSs have small radiation power with low altitude antennas, the interference from the SBS to the MUs or the SUs of the other small cells (SC) can be neglected. The received signal in the $k^{t h} \mathrm{SU}$ of the $s^{t h} \mathrm{SBS}$ is written as:

$$
\begin{aligned}
y_{k, s}^{S} & =\left(\mathbf{h}_{k, s}^{S}\right)^{H} \mathbf{v}_{k, s}^{S} x_{k, s}^{S}+\sum_{\substack{i=1 \\
i \neq k}}^{K}\left(\mathbf{h}_{k, s}^{S}\right)^{H} \mathbf{v}_{i, s}^{S} x_{i, s}^{S} \\
& +\sum_{l=1}^{M}\left(\mathbf{h}_{k, s}^{M S}\right)^{H} \mathbf{v}_{l}^{M} x_{l}^{M}+n_{k, s}
\end{aligned}
$$

$\mathbf{h}_{k, s}^{S} \in C^{N_{s} \times 1}$ is the channel from the $s^{t h}$ SBS to its $k^{t h}$ SU. $\mathbf{h}_{k, s}^{M S} \in C^{N_{m} \times 1}$ is the channel vector from the MBS to the $k^{t h} \mathrm{SU}$ of the $s^{t h}$ small cell. $\mathbf{v}_{i, s}^{S}$ and $x_{i, s}^{S}$ represent the precoding vector and the transferred signal from the $s^{\text {th }}$ SBS toward its $i^{t h} \mathrm{SU}$. The first term in $(2)$ is the desired signal received by the $k^{t h}$ SU. The second and third terms are the interferences from the corresponding SBS and the MBS to the $k^{t h} \mathrm{SU}$, respectively. The data rate of the $m^{\text {th }} \mathrm{MU}$ can be written as $r_{m}=\log _{2}\left(1+\gamma_{m}\right)(\mathrm{bit} / \mathrm{s} / \mathrm{Hz})$, where $\gamma_{m}$ is the SINR on the $m^{\text {th }}$ MU side as:

$$
\gamma_{m}=\frac{\left|\left(\mathbf{h}_{m}^{M}\right)^{H} \mathbf{v}_{m}^{M}\right|^{2}}{\sum_{l \neq m}^{M}\left|\left(\mathbf{h}_{m}^{M}\right)^{H} \mathbf{v}_{l}^{M}\right|^{2}+\sigma_{m}^{2}}
$$

Note that in (3), the intercell interference is disregarded because the transmitting power of the SBS is lower than that of the MBS. Besides the total power consumption consists of two parts: transmission power and circuit power. For heterogeneous downlink scenario, the total power can be calculated as $P_{\text {Tra }}+P_{C i r}$ 
where the transmission power $P_{T r a}$ and circuit power $P_{C i r}$ terms are [21], 13]:

$$
\begin{aligned}
& P_{T r a}=\rho_{0} \sum_{m \in M} \operatorname{Tr}\left(\mathbf{v}_{m}^{M} \mathbf{v}_{m}^{M}{ }^{H}\right)+\sum_{s \in S} \rho_{s} \sum_{k \in K} \operatorname{Tr}\left(\mathbf{v}_{k, s}^{S} \mathbf{v}_{k, s}^{S}{ }^{H}\right) \\
& P_{C i r}=\frac{\eta_{0}}{C} N_{m}+\sum_{s=1}^{S} \frac{\eta_{s}}{C} N_{s}
\end{aligned}
$$

where $\rho_{0}, \rho_{s}$ are the amplifier power efficiency factors of MBS and SBS, respec-

tively. In the circuit power term, $\eta_{0}$ and $\eta_{s}$ denote the power dissipation in the circuit of each transmitter antenna in the MBS and SBS, respectively. $C$ represents the number of subcarriers $(C \geq 1)$ applied to normalize the circuit power.

\section{The Proposed Method}

In this section, the proposed method is illustrated. We would design a precoding matrix to mitigate the interference at the same time of ensuring a minimum data rate as well as a constraint on the maximum transmision power in the presence of imperfect CSI. Due to the large transmission power and coverage radius of the MBS, it causes strong interference in the MUs and SUs. We try to limit this interference through designing an optimal precoding matrix in the MBS. Suppose that $\mathbf{v}_{l}^{M}$ is the precoding vector of the $l^{\text {th }}$ MU. The desired signal of the $l^{\text {th }}$ MU shall be precoded in a way that its interference to any other MUs and SUs be zero. In other words, in order to nullify the second term in (1) and the third term in 22, we shall have:

$$
\begin{aligned}
\left(\mathbf{h}_{m}^{M}\right)^{H} \mathbf{v}_{l}^{M}=0 & \forall l \neq m \\
\left(\mathbf{h}_{k, s}^{M S}\right)^{H} \mathbf{v}_{l}{ }^{M}=0 & \forall k
\end{aligned}
$$

We can rewrite the constraints in (5) in the matrix form as:

$$
\overline{\mathbf{H}}_{l}^{H} \mathbf{v}_{l}^{M}=0
$$

where $\overline{\mathbf{H}}_{l} \in C^{N_{m} \times(M-1+S K)}$ is the interference channel matrix of the $l^{\text {th }}$ MU defined as:

$$
\overline{\mathbf{H}}_{l}=\left[\mathbf{h}_{1}^{M}, \mathbf{h}_{2}^{M}, \ldots, \mathbf{h}_{l-1}^{M}, \mathbf{h}_{l+1}^{M}, \ldots, \mathbf{h}_{M}^{M}, \mathbf{h}_{1,1}^{M S}, \ldots, \mathbf{h}_{K, S}^{M S}\right]
$$


The constraint in (6) removes the interference from the MBS to the other MUs and SUs. Moreover, in order to eliminate the interference from the SBS to the other SUs, the common ZF precoder is applied in the SBS. In other words, in the proposed method, three types of interference in a HetNet have been eliminated. The interference between MUs and the inter-tier interference from the MBS to SUs have been mitigated with the optimal precoding matrix of the MBS. Also, the ZF precoder is applied to remove the intra-cell interference of the small cells. Due to the arrangement of the small cells within the macro cell and the limitted power and low altitude of the SBSs, the interference from SBSs to the MUs can be neglected.

In most of the works in the literature, the precoding matrix is designed based on the assumption of perfect CSI. However, due to the density of users and SBSs in the massive MIMO HetNet, this assumption is not reasonable and the channel estimation is done erroneously. As a result, to have a more realistic modeling, we assume that imperfect CSI is available in the transmitter side. With this in mind, we consider a channel estimation error vector for each user. Hence, the perfect channel vector for the $m^{t h}$ user is modeled as follows:

$$
\mathbf{h}_{m}^{M}=\widehat{\mathbf{h}}_{m}^{M}+\mathbf{e}_{m}
$$

where $\widehat{\mathbf{h}}_{m}^{M}$ is the estimated channel vector and $\mathbf{e}_{m}$ is the estimation error vector. Similarly, the accurate interference channel matrix of the $m^{\text {th }} \mathrm{MU}, \overline{\mathbf{H}}_{m}$, can be represented as:

$$
\overline{\mathbf{H}}_{m}=\widehat{\mathbf{H}}_{m}+\mathbf{E}_{m}
$$

where $\mathbf{E}_{m}=\left[\mathbf{e}_{1}, \mathbf{e}_{2}, \ldots, \mathbf{e}_{m-1}, \mathbf{e}_{m+1}, \ldots, \mathbf{e}_{M}, \mathbf{e}_{1,1}^{M S}, \ldots, \mathbf{e}_{K, S}^{M S}\right]$ is the corresponding estimation error matrix, and $\widehat{\mathbf{H}}_{m}$ is the corresponding estimated interference channel matrix. Using (9), the interference suppression term in (6) can be rewritten as:

$$
\left(\widehat{\mathbf{H}}_{m}+\mathbf{E}_{m}\right)^{H} \mathbf{v}_{m}^{M}=0
$$

Moreover, in the modeling of the channel estimation error, we assume that only a few of the user channel vectors have been erroneously estimated. Hence, only a 
few of the rows of the error matrix, $\mathbf{E}_{m}$, would be non-zero which yields the joint sparsity structure for this matrix 22], 223. To impose the joint sparsity property on a matrix, its mixed $L_{2} / L_{0}$ norm is minimized $\left(\left\|\mathbf{E}_{m}\right\|_{2,0}\right)$. In other words, the $L_{2}$ norm of the matrix rows are calculated, then the $L_{0}$ norm of those $L_{2}$ norms are obtained. This value indicates the number of non-zero rows of a matrix. However, the mixed $L_{2} / L_{0}$ norm is not convex; thus, its convex relaxation which is the mixed $L_{2} / L_{1}$ norm would be minimized $\left(\left\|\mathbf{E}_{m}\right\|_{2,1}\right)$. Considering the interference suppression term as well as the joint sparsity property of the error matrix, we can write the optimization problem for the optimal precoder design as follows:

$$
\begin{array}{ll}
\min _{\mathbf{v}_{m}^{M}, \mathbf{E}_{m}, \mathbf{e}_{m}} & \left\|\left(\widehat{\mathbf{H}}_{m}+\mathbf{E}_{m}\right)^{H} \mathbf{v}_{m}^{M}\right\|_{2}^{2}+\lambda\left\|\mathbf{E}_{m}\right\|_{2,1} \quad \forall m \in M \\
\text { s.t } \quad & C_{1}:\left\|\mathbf{v}_{m}^{M}\right\|_{2}^{2} \leq \frac{P_{\max }}{M} \\
& C_{2}:\left\|\left(\widehat{\mathbf{h}}_{m}^{M}+\mathbf{e}_{m}\right)^{H} \mathbf{v}_{m}^{M}\right\|_{2}^{2}-\gamma_{\min } \sum_{l \neq m}\left\|\left(\widehat{\mathbf{h}}_{m}^{M}+\mathbf{e}_{m}\right)^{H} \mathbf{v}_{l}^{M}\right\|_{2}^{2} \geq \gamma_{\min } \sigma_{m}^{2}
\end{array}
$$

where $\|\cdot\|_{2}$ indicates for the $L_{2}$ norm of the vector and $\lambda$ is the relaxation parameter which controls the effect of the joint sparsity term. The constraint $\left(C_{1}\right)$ is to guarantee the maximum transmission power of the MBS to each of 90 the MUs. $P_{\max }$ is the maximum transmission power of the MBS. The constraint $\left(C_{2}\right)$ ensures the MUs to have SINR higher than $\gamma_{\min }$. As a result, this constraint guarantees a minimum data rate of $\log _{2}\left(1+\gamma_{\text {min }}\right)$ transmitted for each macro user.

Note that the optimization problem in 111 is non-convex due to the coupling of the optimization variables in the cost function and the $C_{2}$ constraint. Hence, the block coordinate descent (BCD) algorithm in 24] is adopted to evaluate the convexity of the problem with respect to each of variables. The main idea of the coordinate descent algorithm is to alternately optimize the problem with respect to one block of the variables while the others are fixed. In the convexity analysis of the problem (11) with respect to each of the variables separately, the problem 111 is convex in respect of the variable $\mathbf{E}_{m}$, while the other two subproblems with respect to $\mathbf{v}_{m}^{M}$, and $\mathbf{e}_{m}$ are non-convex due to the non-convexity 
of the $C_{2}$ constraint. Indeed, if the BCD method is applied to the problem (11) in its current form, the optimization problem will still be non-convex. Hence, in order to tackle the non-convexity and also unify the variables in the whole problem, the $C_{2}$ constraint and the cost function are rewritten as:

$$
\begin{array}{r}
\left\|\left(\widehat{\mathbf{h}}_{m}^{M}+\mathbf{e}_{m}\right)^{H} \mathbf{v}_{m}^{M}\right\|_{2}^{2}-\gamma_{\min } \sum_{l \neq m}\left\|\left(\widehat{\mathbf{h}}_{m}^{M}+\mathbf{e}_{m}\right)^{H} \mathbf{v}_{l}^{M}\right\|_{2}^{2}= \\
\left.\operatorname{Tr}\left(\mathbf{W}_{m}-\gamma_{\min } \sum_{l \neq m} \mathbf{W}_{l}\right)\left(\widehat{\mathbf{h}}_{m}^{M}\left(\widehat{\mathbf{h}}_{m}^{M}\right)^{H}+2 \operatorname{Re}\left\{\widehat{\mathbf{h}}_{m}^{M} \mathbf{e}_{m}^{H}\right\}+\mathbf{e}_{m} \mathbf{e}_{m}^{H}\right)\right\}
\end{array}
$$

and

$$
\left\|\left(\widehat{\mathbf{H}}_{m}+\mathbf{E}_{m}\right)^{H} \mathbf{v}_{m}^{M}\right\|_{2}^{2}=\operatorname{Tr}\left\{\mathbf{W}_{m}\left(\widehat{\mathbf{H}}_{m} \widehat{\mathbf{H}}_{m}^{H}+\widehat{\mathbf{H}}_{m} \mathbf{E}_{m}^{H}+\mathbf{E}_{m} \widehat{\mathbf{H}}_{m}^{H}+\mathbf{E}_{m} \mathbf{E}_{m}^{H}\right)\right.
$$

where $\operatorname{Tr}\{$.$\} indicates for the trace of the matrix and \mathbf{W}_{m} \triangleq \mathbf{v}_{m}^{M} \mathbf{v}_{m}^{M^{H}}$ is a positive semidefinite (psd) matrix with $\operatorname{rank}\left(\mathbf{W}_{m}\right)=1$. By lifting the vector $\mathbf{v}_{m}^{M}$ to the matrix $\mathbf{W}_{m}$, the sub-problem with respect to $\mathbf{W}_{m}$ would be convex since the cost function as well as the constraint would be an affine function. However, the sub-problem with respect to $\mathbf{e}_{m}$ is still non-convex since the constraint $C_{2}$ is a concave-convex function. In order to solve this issue, the $C_{2}$ constraint is restated as:

$$
\begin{aligned}
& \left\|\left(\widehat{\mathbf{h}}_{m}^{M}+\mathbf{e}_{m}\right)^{H} \mathbf{v}_{m}^{M}\right\|_{2}^{2}-\gamma_{\min } \sum_{l \neq m}\left\|\left(\widehat{\mathbf{h}}_{m}^{M}+\mathbf{e}_{m}\right)^{H} \mathbf{v}_{l}^{M}\right\|_{2}^{2}= \\
& \operatorname{Tr}\left\{\left(\mathbf{W}_{m}-\gamma_{\min } \sum_{l \neq m} \mathbf{W}_{l}\right)\left(\widehat{\mathbf{h}}_{m}^{M}\left(\widehat{\mathbf{h}}_{m}^{M}\right)^{H}+2 \operatorname{Re}\left\{\widehat{\mathbf{h}}_{m}^{M} \mathbf{e}_{m}^{H}\right\}+\mathbf{e}_{m} \mathbf{e}_{m}^{H}\right)\right\}= \\
& \operatorname{Tr}\left\{\widetilde{\mathbf{e}}_{m}^{H} \boldsymbol{\Xi}^{H} \mathbf{W}_{m} \boldsymbol{\Xi} \widetilde{\mathbf{e}}_{m}\right\}-\gamma_{\min } \sum_{l \neq m} \operatorname{Tr}\left\{\tilde{\mathbf{e}}_{m}^{H} \boldsymbol{\Xi}^{H} W_{l} \boldsymbol{\Xi} \tilde{\mathbf{e}}_{m}\right\}= \\
& \operatorname{Tr}\left\{\boldsymbol{\Gamma}_{m} \boldsymbol{\Xi}^{H} \mathbf{W}_{m} \boldsymbol{\Xi}\right\}-\gamma_{\min } \sum_{l \neq m} \operatorname{Tr}\left\{\boldsymbol{\Gamma}_{m} \boldsymbol{\Xi}^{H} \mathbf{W}_{l} \boldsymbol{\Xi}\right\}
\end{aligned}
$$

where $\boldsymbol{\Xi}=\left[\mathbf{I}, \widehat{\mathbf{h}}_{m}\right], \widetilde{\mathbf{e}}_{m}=\left[\mathbf{e}_{m}, x\right]^{H}$ and $\boldsymbol{\Gamma}_{m}=\widetilde{\mathbf{e}}_{m} \widetilde{\mathbf{e}}_{m}^{H}$ is a positive semidefinite (psd) matrix with rank one. In other words, the varaible vector $\mathbf{e}_{m}$ is lifted to the matrix $\boldsymbol{\Gamma}_{m}$. By replacing (14) and (13), (11) can be written in the following 
equivalent form:

$$
\begin{aligned}
& \min _{\mathbf{W}_{m}, \mathbf{E}_{m}, \boldsymbol{\Gamma}_{m}} \operatorname{Tr}\left\{\mathbf{W}_{m}\left(\widehat{\mathbf{H}}_{m} \mathbf{H}_{m}^{H}+\widehat{\mathbf{H}}_{m} \mathbf{E}_{m}^{H}+\mathbf{E}_{m} \widehat{\mathbf{H}}_{m}^{H}+\mathbf{E}_{m} \mathbf{E}_{m}^{H}\right)\right\} \\
& \quad+\lambda\left\|\mathbf{E}_{m}\right\|_{2,1} \quad \forall m \in M \\
& \text { s.t } \quad C_{1}: \operatorname{Tr}\left\{\mathbf{W}_{m}\right\} \leq \frac{P_{\max }}{M} \\
& C_{2}: \operatorname{Tr}\left\{\boldsymbol{\Gamma}_{m} \boldsymbol{\Xi}^{H} \mathbf{W}_{m} \boldsymbol{\Xi}\right\}-\gamma_{\min } \sum_{l \neq m} \operatorname{Tr}\left\{\boldsymbol{\Gamma}_{m} \boldsymbol{\Xi}^{H} \mathbf{W}_{l} \boldsymbol{\Xi}\right\} \geq \gamma_{\min } \sigma_{m}^{2} \\
& C_{3}: \mathbf{W}_{m} \in S_{n}^{+} \quad C_{4}: \boldsymbol{\Gamma}_{m} \in S_{n}^{+} \\
& C_{5}: \operatorname{rank}\left(\mathbf{W}_{m}\right)=1 \quad C_{6}: \operatorname{rank}\left(\boldsymbol{\Gamma}_{m}\right)=1
\end{aligned}
$$

The problem 15 is still non-convex due to the coupling of the optimization variables in the cost function and $C_{2}$ as well as the presence of rank-one constraints in $C_{5}$ and $C_{6}$. By applying the semidefinite relaxation (SDR) method [25], we get rid of the rank-one constraints. Next, to deal with non-convexity, we separate the variables into two blocks $\left(\left\{\mathbf{W}_{m}\right\},\left\{\mathbf{E}_{m}, \boldsymbol{\Gamma}_{m}\right\}\right)$. Therefore, the problem becomes convex with respect to each block of variables when the other one is fixed. We apply the BCD technique to solve the problem (15). At the sub-problem of $\mathbf{W}_{m}$, the variables $\left\{\mathbf{E}_{m}\right.$ and $\left.\boldsymbol{\Gamma}_{m}\right\}$ are assumed as fixed and the problem is solved with respect to $\mathbf{W}_{m}$ :

$$
\begin{array}{ll}
\left.\min _{\mathbf{W}_{m}} \operatorname{Tr} \mathbf{W}_{m}\left(\widehat{\mathbf{H}}_{m} \mathbf{H}_{m}^{H}+\widehat{\mathbf{H}}_{m} \mathbf{E}_{m}^{H}+\mathbf{E}_{m} \widehat{\mathbf{H}}_{m}^{H}+\mathbf{E}_{m} \mathbf{E}_{m}^{H}\right)\right\} \quad \forall m \in M \\
\text { s.t } \quad C_{1}: \operatorname{Tr}\left\{\mathbf{W}_{m}\right\} \leq \frac{P_{\max }}{M} \\
C_{2}: \operatorname{Tr}\left\{\boldsymbol{\Gamma}_{m} \boldsymbol{\Xi}^{H} \mathbf{W}_{m} \boldsymbol{\Xi}\right\}-\gamma_{\min } \sum_{l \neq m} \operatorname{Tr}\left\{\boldsymbol{\Gamma}_{m} \Xi^{H} \mathbf{W}_{l} \boldsymbol{\Xi}\right\} \geq \gamma_{\min } \sigma_{m}^{2} \\
C_{3}: \mathbf{W}_{\mathbf{m}} \in S_{n}^{+}
\end{array}
$$

16) is a SemiDefinite Programming (SDP) problem which can be solved by the CVX optimization toolbox [26] or any other SDP solvers. At the second sub-problem, $\mathbf{W}_{m}$ is fixed and the problem is solved with respect to $\left\{\mathbf{E}_{m}, \boldsymbol{\Gamma}_{m}\right\}$. 
Thus, the second optimization sub-problem as:

$$
\begin{aligned}
\min _{\mathbf{E}_{m}, \boldsymbol{\Gamma}_{m}}( & \left.\operatorname{Tr}\left\{\mathbf{W}_{m}\left(\widehat{\mathbf{H}}_{m} \mathbf{H}_{m}^{H}+\widehat{\mathbf{H}}_{m} \mathbf{E}_{m}^{H}+\mathbf{E}_{m} \widehat{\mathbf{H}}_{m}^{H}+\mathbf{E}_{m} \mathbf{E}_{m}^{H}\right)\right\}\right) \\
& \left.+\lambda\left\|\mathbf{E}_{m}\right\|_{2,1}\right) \quad \forall m \in M \\
\text { s.t } \quad C_{1}: & \operatorname{Tr}\left\{\boldsymbol{\Gamma}_{m} \boldsymbol{\Xi}^{H} \mathbf{W}_{m} \boldsymbol{\Xi}\right\}-\gamma_{\min } \sum_{l \neq m} \operatorname{Tr}\left\{\boldsymbol{\Gamma}_{m} \boldsymbol{\Xi}^{H} \mathbf{W}_{l} \boldsymbol{\Xi}\right\} \geq \gamma_{\min } \sigma_{m}^{2} \\
& C_{2}: \boldsymbol{\Gamma}_{\mathbf{m}} \in S_{n}^{+}
\end{aligned}
$$

The problem (17) is convex and can be solved using the CVX optimization toolbox [26].

The beamforming vector can be estimated from the sub-optimal matrix $\mathbf{W}_{m}$ by applying the eigen value decomposition (EVD) as: $\mathbf{v}_{m}^{M}=\mathbf{u} \xi^{\frac{1}{2}}$ where $\xi$ is the largest eigenvalue of $\mathbf{W}_{m}$ and $\mathbf{u}$ is its corresponding eigenvector. The same is done for extracting $\widetilde{\mathbf{e}}_{m}$ from the $\boldsymbol{\Gamma}_{m}$ matrix. Therefore, by applying the EVD on the obtained $\boldsymbol{\Gamma}_{m}$ matrix, we will have: $\boldsymbol{\Gamma}_{m}=\mathbf{U} \boldsymbol{\Sigma} \mathbf{U}^{H}$ where $\mathbf{U}$ is the unitary matrix of the eigen vectors and $\boldsymbol{\Sigma}$ is the diagonal matrix of the eigenvalues. In order to guarantee the rank-one constraint on $\boldsymbol{\Gamma}_{m}$, the gaussian randomization technique in [27] is adopted to produce a suboptimal solution of $\widetilde{\mathbf{e}}_{m}$ as $\widetilde{\mathbf{e}}_{m}=\mathbf{U} \boldsymbol{\Sigma}^{\frac{1}{2}} \mathbf{r}$ where $\mathbf{r} \sim C N\left(\mathbf{0}_{N_{m}+1}, \mathbf{I}_{N_{m}+1}\right)$ is a random vector with circularly symmetric complex Gaussian (CSCG) distribution. Finally, the suboptimal solution of $\mathbf{e}_{m}$ is given as:

$$
\mathbf{e}_{m}=\frac{\left[\tilde{\mathbf{e}}_{m}\right]_{\left(1: N_{M}\right)} /\left[\tilde{\mathbf{e}}_{m}\right]_{\left(N_{M+1}\right)}}{\left\|\left[\tilde{\mathbf{e}}_{m}\right]_{\left(1: N_{M}\right)} /\left[\tilde{\mathbf{e}}_{m}\right]_{\left(N_{M+1}\right)}\right\|_{2}}
$$

and $[\mathbf{z}]_{(1: L)}$ refers to the vector that contains the first $L$ elements of $\mathbf{z}$.

The proposed method has been summarized in Algorithm 1 .

\section{NUMERICAL RESULTS}

In this section, the performance of the suggested algorithm has been investigated through various simulation scenarios. The considered system is a macro cell with a radius of $500 \mathrm{~m}$ that includes one MBS located at the center of the 


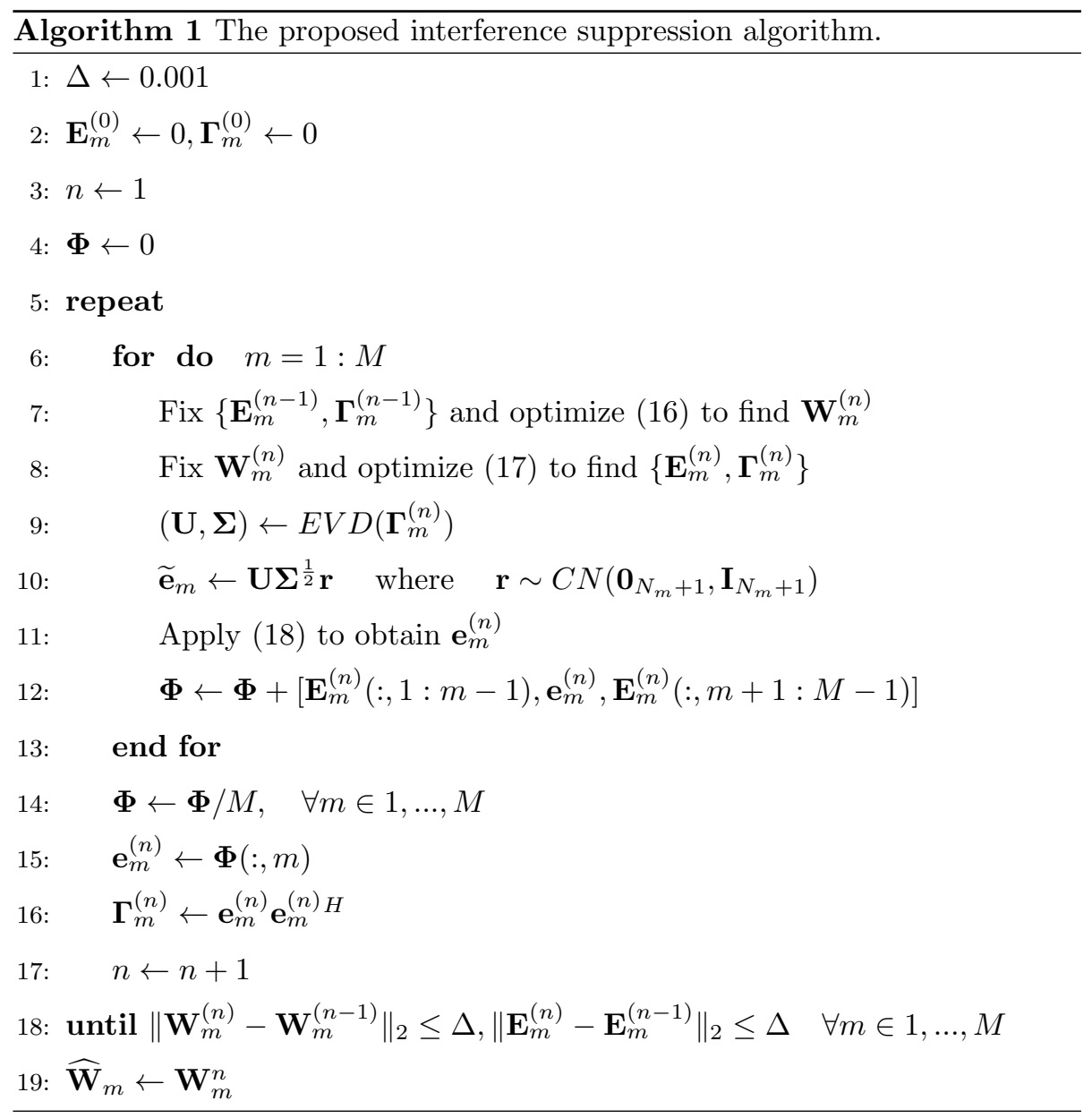


macro-cell with 15 MUs. Also, there are 5 SCs where each SBS serves three SUs within a 40m range. The channel is modeled as follows [13]: $\mathbf{h}_{m}^{M}=\sqrt{\varphi_{m}^{M}} \mathbf{g}_{m}^{M}$ where $\mathbf{g}_{m}^{M} \in C^{1 \times N_{m}}$ is the small scale fading generated from Rayleigh distribution and $\varphi_{m}^{M}$ is the large scale fading which includes the path loss and shadowing.

Throughout the simulations, it is assumed that the SUs are scattered in a cell with a radius much smaller than that of the macro cell. Also, SCs are placed far away from the MBS. Based on these assumptions, the angle of arrival of the signal from MBS to a SBS and its SUs will be almost equal. Thus, it can be claimed that the channel vectors from the MBS to a SBS and its SUs would be highly correlated The simulation parameters are presented in Table 1 . The following methods have been considered as the benchmarks: the Energy-

Table 1: The Simulation Parameters.

\begin{tabular}{ll}
\hline \hline parameters & values \\
\hline Bandwidth & $10 \mathrm{MHz}$ \\
Maximum MBS power budget & $46 \mathrm{dBm}$ \\
The minimum rate required for each user & $6 \mathrm{Mbps}$ \\
Standard deviation of shadowing & $7 \mathrm{~dB}$ \\
The least distance of the SBSs from the MBS & $150(\mathrm{~m})$ \\
MBS penetration loss & $128.1+37.6 \log _{10}(d(\mathrm{~km}))$ \\
SBS penetration loss & $127+30 \log _{10}(d(\mathrm{~km}))$ \\
\hline
\end{tabular}

140

Efficient Zero-Forcing HetNet Beamforming (EE ZF) technique [16] and the All-BS method [15].

The performance of the proposed precoding matrix is evaluated from the perspective of the MBS transmission power, sum rate $\left(\sum_{m} r_{m}+\sum_{s} \sum_{k} r_{k, s}\right)$, and 145 energy efficiency. 30 Monte Carlo runs on the channel matrix have been conducted in all of the simulation scenarios. Furthermore, it is assumed that only $5 \mathrm{MU}$ channels (which are selected uniformly at random) have been erroneously estimated. In the first test, the effect of the parameter $\lambda$ has been investigated. 


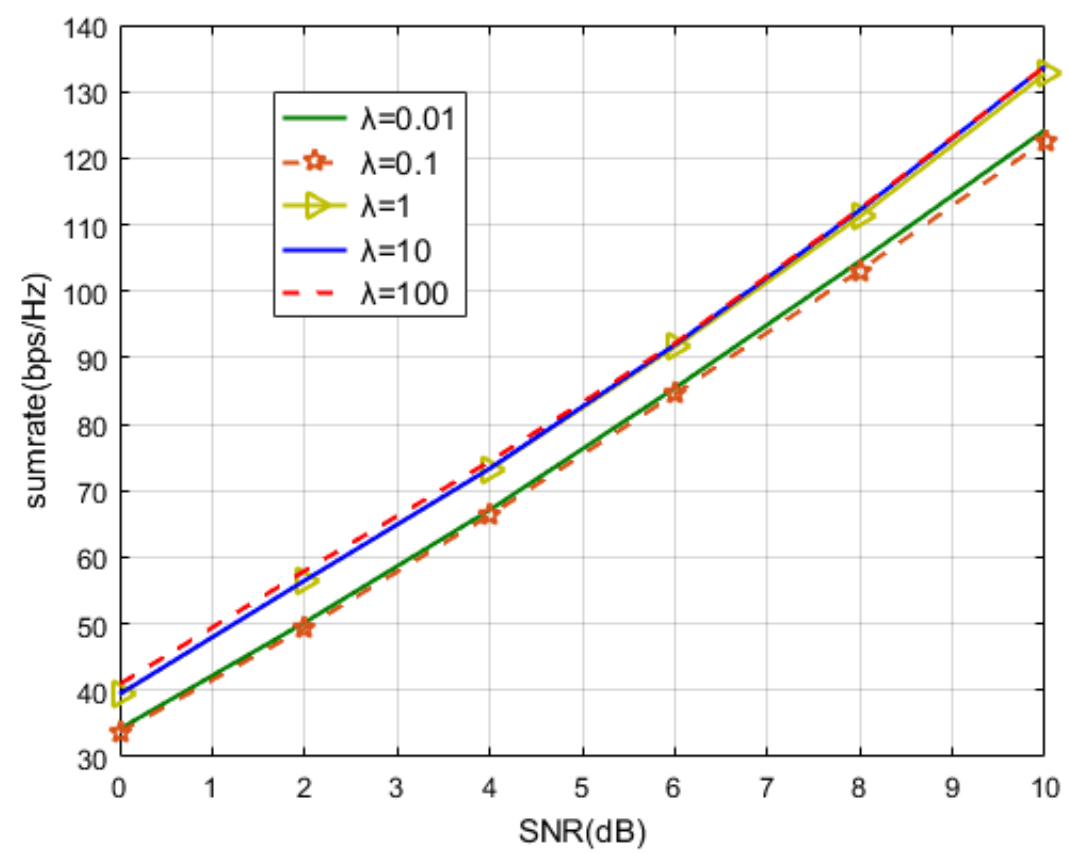

Figure 2: The sum-rate versus SNR curves for different values of $\lambda$.

Fig 2 depicts the sum-rate versus SNR curves for various values of the parameter $\lambda$. The number of transmit antennas has been considered as 40 in this simulation.

As the amount of $\lambda$ increases from 0.01 to 1 , the sum-rate increases and for the $\lambda$ values greater than 1 , the sum-rate curve does not change. Therefore, we have set $\lambda=1$ for the rest of the simulations.

In the following, we compare the efficiency of the provided precoder with the other competing methods. Fig 3 shows the sum-rate versus SNR curves for all the 3 methods.

According to the figure, the sum-rate curves have increasing trend with respect to SNR for all of the three methods. The proposed precoding scheme offers higher sum-rate for all SNR values. The outperformance of the proposed scheme is more considerable in high-SNR regimes. This is due to the fact that the proposed scheme has modeled the channel estimation error as well as sup- 


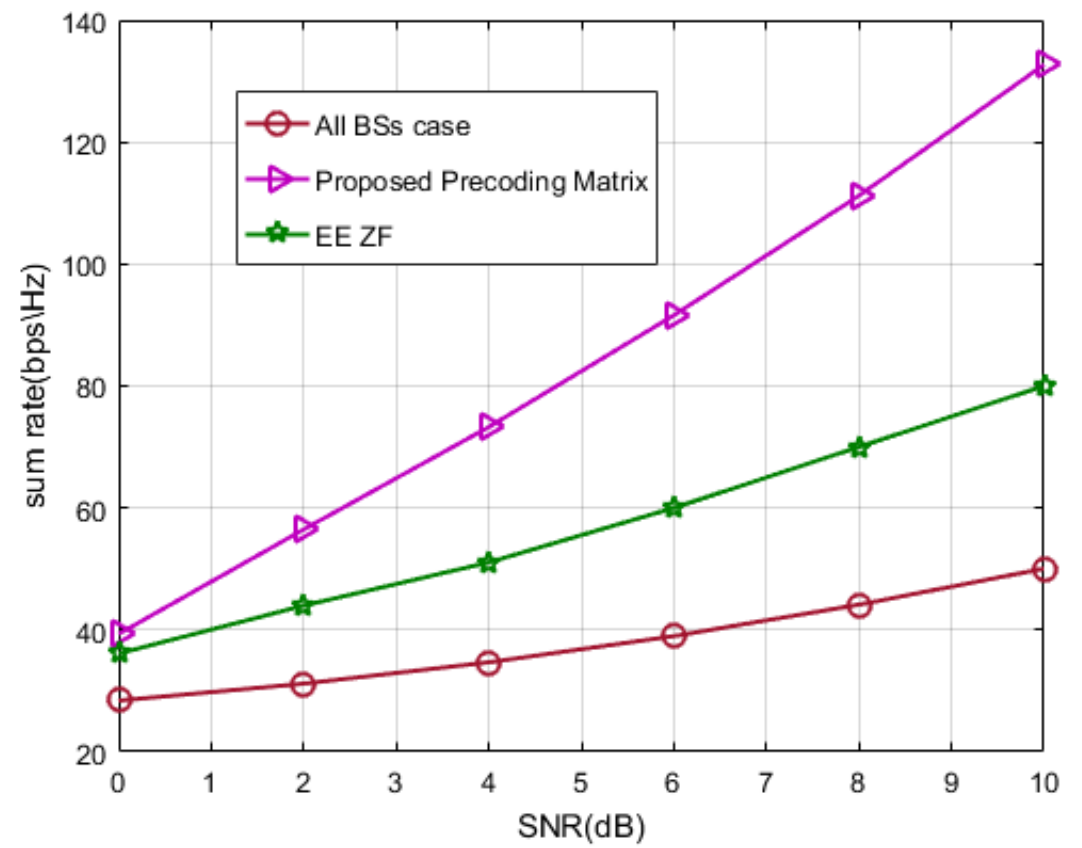

Figure 3: The comparison of sum-rate versus SNR curves for all the methods with 40 transmission antennas. 


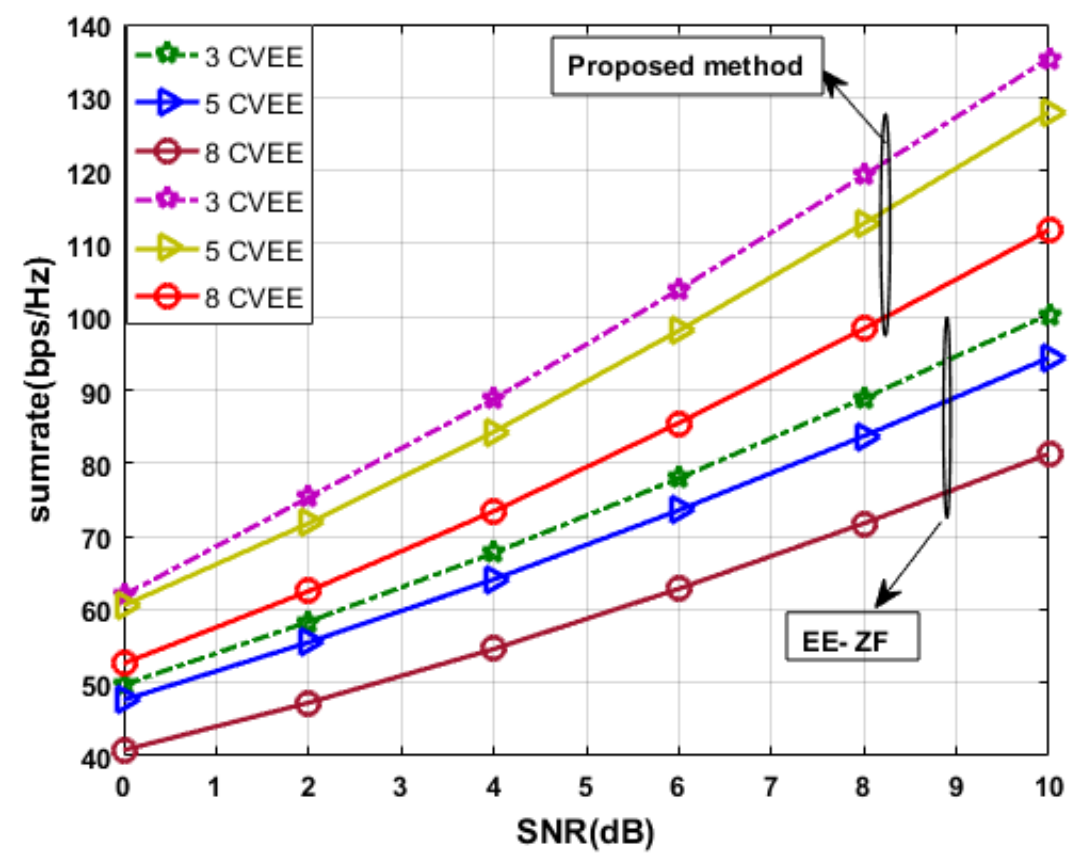

Figure 4: The sum-rate versus SNR for different EECV with 60 antennas.

pressing the interference.

It should be noted that the All-BS method has very high computational complexity for large antenna array size. Therefore, it could not be compared in the rest of the simulations where the antenna number is greater than 40 . In the next scenario, the effect of the number of erroneously estimated channel vectors (EECV) has been investigated.

Figure 4 shows the sum-rate versus SNR curves for various numbers of erroneously estimated channels with 60 transmission antennas.

As expected, the sum-rate increases with the SNR growth. According to the figure 4, the transmission rate decreases as the number of incorrect channels increases. Furthermore, the proposed method achieves higher sum-rate even in the case of erroneous channel estimation.

In Figure 5, the transmission power curves have been depicted with respect to the number of MBS antennas. 


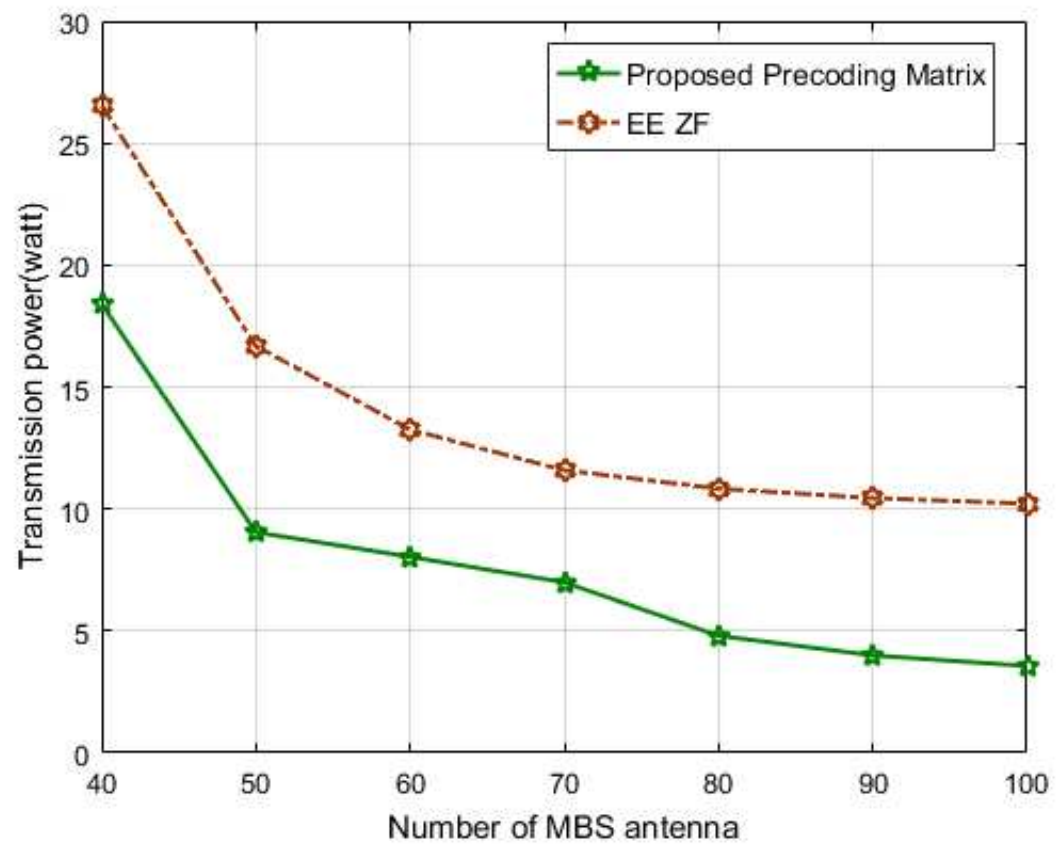

Figure 5: The transmission power versus the number of MBS antennas. 


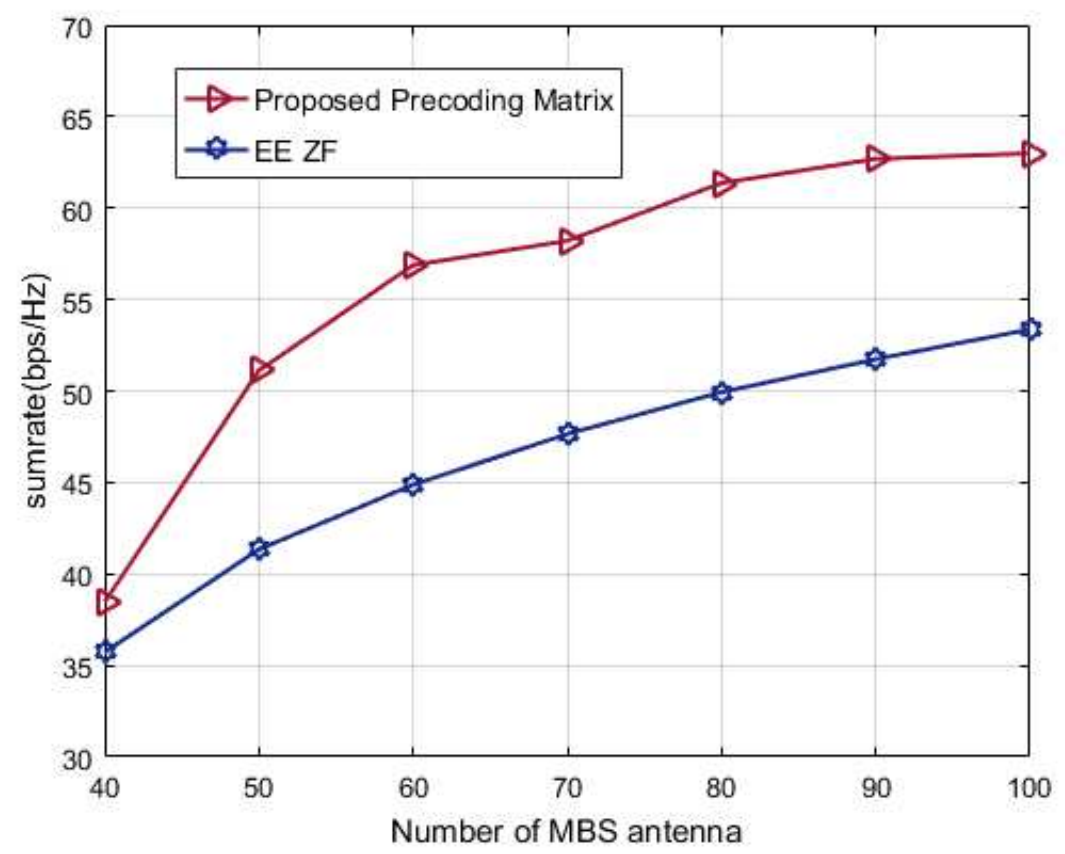

Figure 6: The sum-rate versus the number of MBS antennas.

For both of the methods, the transmission power decreases as the number of antennas increases. In addition, the proposed method has lower transmission power than the EE-ZF scheme.

Fig 6 displays the curves of the sum-rate versus the number of transmit antennas.

It is observed that the sum-rate increases for the larger antenna sizes which indicates the advantage of the massive MIMO systems. Furthermore, the proposed method acheives higher sum-rate values compared to the EE-ZF scheme specially in the larger antenna sizes. Meanwhile, the gap between the transmission rates of the two methods depends on the capability of the precoder in eliminating the interference in the presence of channel estimation error. Our proposed method performs better in such situations due to the modeling of channel estimation error in the precoder matrix design.

As another scenario, the energy efficiency curves versus the number of trans- 


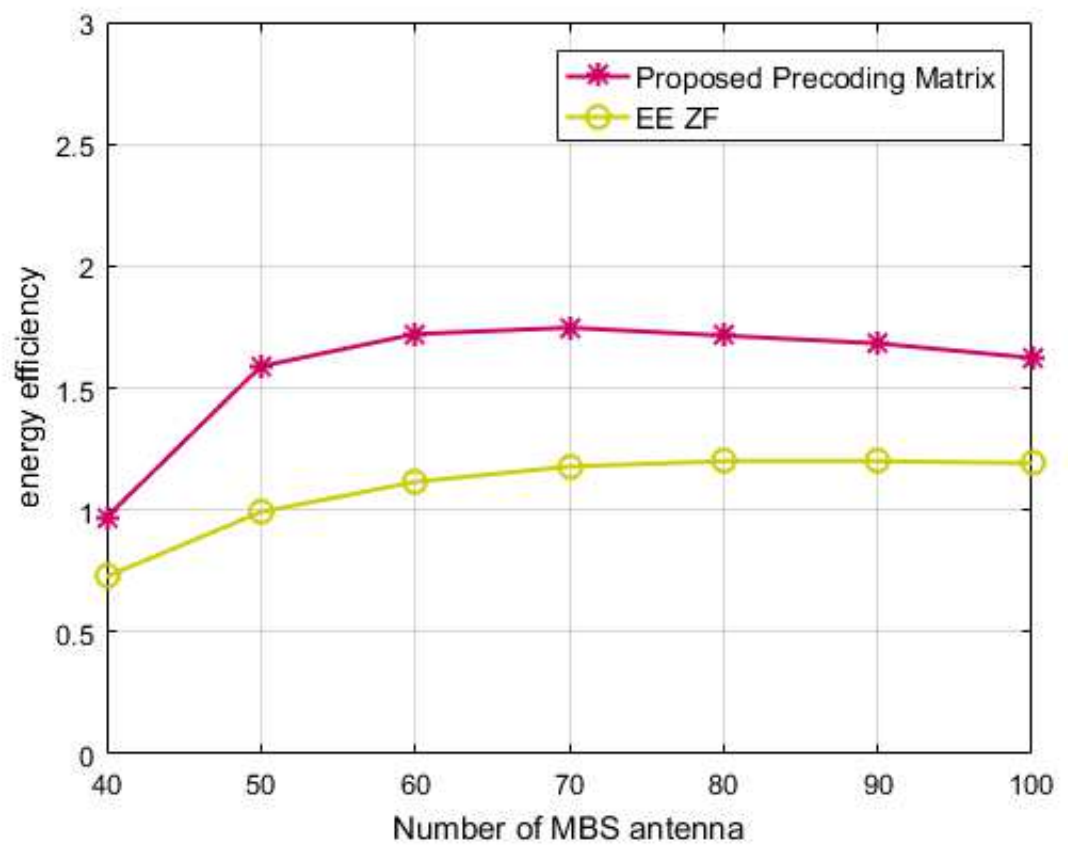

Figure 7: The energy efficiency versus the number of transmit antennas.

mit antennas have been plotted in Figure 7

According to this figure, the energy efficiency is increasing for less than 70 antennas. This is because of the increasing trend of the spectral efficiency in this range. As the transmit antennas goes beyond 70, the inter-user interference and the circuit power of the MBS increase as well which leads to a slight decrease in the energy efficiency. The proposed method outperforms the EE-ZF scheme even in the case of energy efficiency.

\section{CONCLUSION}

In this paper, a MIMO precoding scheme has been suggested to cope with the interference of the massive MIMO HetNets. The channel estimation error has been modeled in the proposed scheme under the assumption that a few of the channel estimations are imperfect. The simulation results indicate that the 
proposed method outperforms its rivals in sum rate and energy efficiency.

\section{Conflict of Interest}

205 conflict of interest.

\section{References}

[1] J. G. Andrews, S. Buzzi, W. Choi, S. V. Hanly, A. Lozano, A. C. Soong, J. C. Zhang, What will 5g be?, IEEE Journal on selected areas in communications 32 (6) (2014) 1065-1082.

[2] T. K. Vu, S. Kwon, S. Oh, Cooperative interference mitigation algorithm in heterogeneous networks, IEICE Transactions on Communications 98 (11) (2015) 2238-2247.

[3] A. He, L. Wang, Y. Chen, K.-K. Wong, M. Elkashlan, Uplink interference management in massive mimo enabled heterogeneous cellular networks, IEEE Wireless Communications Letters 5 (5) (2016) 560-563.

[4] Q. Niu, Z. Zeng, T. Zhang, Q. Gao, S. Sun, Joint interference alignment and power allocation in heterogeneous networks, in: 2014 IEEE 25th Annual International Symposium on Personal, Indoor, and Mobile Radio Communication (PIMRC), IEEE, 2014, pp. 733-737.

[5] M. Morales-Céspedes, J. Plata-Chaves, D. Toumpakaris, S. A. Jafar, A. G. Armada, Cognitive blind interference alignment for macro-femto networks, IEEE Transactions on Signal Processing 65 (19) (2017) 5121-5136.

[6] M. Feng, S. Mao, Interference management in massive mimo hetnets: A nested array approach, in: 2016 IEEE Global Communications Conference (GLOBECOM), IEEE, 2016, pp. 1-6. 
[7] Y. Zhang, Y. J. A. Zhang, Adaptive inter-cell interference cancellation in heterogeneous networks: Making smart use of multiple antennas at base stations, in: 2015 IEEE/CIC International Conference on Communications in China (ICCC), IEEE, 2015, pp. 1-6.

[8] R. Peng, Y. Tian, Inter-tier interference coordination in massive-mimo system based on statistical channel information, in: 2016 IEEE/CIC International Conference on Communications in China (ICCC Workshops), IEEE, 2016, pp. 1-6.

235 [9] D. Ying, H. Yang, T. L. Marzetta, D. J. Love, Heterogeneous massive mimo with small cells, in: 2016 IEEE 83rd Vehicular Technology Conference (VTC Spring), IEEE, 2016, pp. 1-5.

[10] T. K. Vu, M. Bennis, S. Samarakoon, M. Debbah, M. Latva-Aho, Joint load balancing and interference mitigation in $5 \mathrm{~g}$ heterogeneous networks, IEEE Transactions on Wireless Communications 16 (9) (2017) 6032-6046.

[11] Y. Liu, L. Lu, G. Li, Q. Cui, Performance analysis and interference cancellation for heterogeneous network with massive mimo, in: 2015 IEEE Global Conference on Signal and Information Processing (GlobalSIP), IEEE, 2015, pp. $888-892$.

[12] S. Bu, F. R. Yu, H. Yanikomeroglu, Interference-aware energy-efficient resource allocation for ofdma-based heterogeneous networks with incomplete channel state information, IEEE Transactions on Vehicular Technology 64 (3) (2014) 1036-1050.

[13] E. Björnson, M. Kountouris, M. Debbah, Massive mimo and small cells: Improving energy efficiency by optimal soft-cell coordination, arXiv preprint arXiv:1304.0553.

[14] Z. Xu, C. Yang, G. Y. Li, Y. Liu, S. Xu, Energy-efficient comp precoding in heterogeneous networks, IEEE Transactions on Signal Processing 62 (4) (2014) 1005-1017. 
[15] L. D. Nguyen, T. Q. Duong, D. N. Nguyen, L.-N. Tran, Energy efficiency maximization for heterogeneous networks: A joint linear precoder design and small-cell switching-off approach, in: 2016 IEEE Global Conference on Signal and Information Processing (GlobalSIP), IEEE, 2016, pp. 718-722.

[16] L. D. Nguyen, H. D. Tuan, T. Q. Duong, O. A. Dobre, H. V. Poor, Downlink beamforming for energy-efficient heterogeneous networks with massive mimo and small cells, IEEE Transactions on Wireless Communications 17 (5) (2018) 3386-3400.

[17] Y. Hao, Q. Ni, H. Li, S. Hou, On the energy and spectral efficiency tradeoff in massive mimo-enabled hetnets with capacity-constrained backhaul links,

[18] S. Ni, J. Zhao, H. H. Yang, T. Q. Quek, Y. Gong, Small cell range expansion with interference mitigation for downlink massive mimo hetnets, in: 2018 IEEE Global Communications Conference (GLOBECOM), IEEE, 2018, pp. $1-7$.

[19] R. Sedghi, M. Azghani, Sparsity-based mimo interference suppression technique in the presence of imperfect channel state information, IET Communications 13 (19) (2019) 3201-3206.

[20] M. Azghani, F. Marvasti, Sparse signal processing, in: New perspectives on approximation and sampling theory, Springer, 2014, pp. 189-213.

[21] Y. Shi, J. Zhang, K. B. Letaief, Group sparse beamforming for green cloud radio access networks, in: 2013 IEEE Global Communications Conference (GLOBECOM), IEEE, 2013, pp. 4662-4667.

[22] N. Sadeghi, M. Azghani, Multi-user massive mimo channel estimation using joint sparsity and non-ideal feedback modeling, Digital Signal Processing 280 $100(2020) 102640$.

[23] F. Afkhaminia, M. Azghani, 2d off-grid doa estimation using joint sparsity, IET Radar, Sonar \& Navigation 13 (9) (2019) 1580-1587. 
[24] X. Yu, J.-C. Shen, J. Zhang, K. B. Letaief, Alternating minimization algorithms for hybrid precoding in millimeter wave mimo systems, IEEE Journal of Selected Topics in Signal Processing 10 (3) (2016) 485-500.

[25] Z.-Q. Luo, W.-K. Ma, A. M.-C. So, Y. Ye, S. Zhang, Semidefinite relaxation of quadratic optimization problems, IEEE Signal Processing Magazine 27 (3) (2010) 20-34.

[26] M. Grant, S. Boyd, CVX: Matlab software for disciplined convex programming, version 2.1, http://cvxr.com/cvx (Mar. 2014).

[27] W.-K. Ma, T. N. Davidson, K. M. Wong, Z.-Q. Luo, P.-C. Ching, Quasimaximum-likelihood multiuser detection using semi-definite relaxation with application to synchronous cdma, IEEE transactions on signal processing 50 (4) (2002) 912-922.

\section{Statements Declarations}

This work was supported by Iran National Science Foundation (INSF) under Grant 99000863. 